\title{
A SOCIO-TECHNICAL APPROACH TO BROADBAND DIFFUSION BY SMES
}

Special Issue on: "Using Technology for Innovation and Knowledge Sharing in SMEs: Sociotechnical Approaches" 


\section{Introduction}

SMEs vary in size and nature depending on their area of business. In the same way, their attitudes differ when it comes to the adoption of new technologies. In some cases, SMEs are keen to accept new technologies while in other situations, they are not. There are many reasons why SMEs may decide to adopt new technologies. These reasons could be related to the desire for business growth (Levy et al., 2002). In other instances it could be as a result of a desire for better communications (Chappell et al., 2002).Unfortunately, the diffusion of new technologies to SMEs is not always straightforward and simple. According to Kalakota and Robinson (2001), the main barriers to information technology (IT) and electronic commerce (e-commerce) adoption by SMEs appear to be the managers' unwillingness to take risks when it comes to technological change.

One of such technological changes in recent times is the use of broadband. Prior to using broadband for browsing the Internet, many SMEs used dial-up connection. According to Firth and Kelly (2001), the term broadband has no established definition and varies from country to country. Broadband technology is an umbrella term which covers varying high-speed access technologies including Asymmetric Digital Subscriber Line (ADSL), cable modems, satellite, and Wireless Fixed (Wi-Fi) networks. Broadband provides an 'always on' and faster Internet connection than dial up. It is an emerging technology that promises to improve Internet use because of the speed it provides and constant connection without causing disruption to phone lines. These advantages notwithstanding, there is little research to show if SMEs have used the Internet differently since the introduction of broadband.

Internet usage by SMEs could vary depending on the industry. There are many different reasons SMEs may have for using the Internet such as, Internet presence of their company and email communication. Also activities like online payments, online purchasing, online banking and customer services. According to Saban and Rau (2005), several benefits for SMEs to make use of Internet or e-commerce include reduced cost to business and greater market exposure. Provision of operational, managerial and strategic advantages such as better links between the SMEs and customers and business partners, greater market penetration, more information for decision making, greater access to external resources and expertise are even more reasons for SMEs use of Internet (Jeffcoate et al., 2002). Merlin (2004) in his study of SMEs in the wine industry in Chile and the coffee industry in Costa Rica found that the use of the Internet was found to be very useful for communicating with importers around the world. It also aided easy provision of marketing materials. There was also the benefit of increased turn over and profit for the businesses.

The use of the Internet by SMEs provides them with the opportunity to compete on the same level as larger companies as stated by Sandler and Boggs (2001). Saban and Rau (2005) also highlight the SMEs use of websites to publish company and/or product information and interact with customers in foreign markets. However in contrast, their research found that limited resources prevented SMEs from using the Internet to conduct more sophisticated transactions thus arguing that the Internet allows competition on the same level between SMEs and larger companies only partially.

Using the Internet may be advantageous for SMEs but there is the issue of how SMEs perceive the importance of the Internet as a communication tool. A few studies show the effect of the Internet on SMEs and their business processes but there is little research on broadband Internet use by SMEs in particular. In this study we try to bridge this gap in the literature and examine broadband diffusion and adoption by SMEs. Our aim is to propose and apply a framework examining the different viewpoints of stakeholders in the diffusion of broadband. 
We start by looking at how the innovation diffusion theory may explain technology diffusion phenomena. In previous studies of diffusion of technologies, the innovation diffusion theory as presented mainly by Rogers (1995) has been widely used to understand the reasons behind adoption of innovations. We argue that the diffusion theories are one-sided trying to identify characteristics of the innovation that would make the users adopt the innovation while the perceptions of other stakeholders are not taken into consideration. Thus, we examine the use of social construction of technology (SCOT) to help us examine the different viewpoints involved in the innovation diffusion process. We then investigate these viewpoints in the case of broadband adoption using empirical data.

The paper is structured as follows. In the next section we present literature in the studies of SMEs and Internet use. In section three, we analyse how the diffusion of innovations theory can be supported by SCOT in order to propose our framework. In section four we present our research approach. In section five, the framework is discussed using fieldwork where representatives of the identified social groups were asked to offer their opinions, which are analysed with a view to get a better understanding of broadband diffusion and its use for electronic business (e-business) by SMEs. In section six, we discuss the results and finally in section seven, we conclude and offer recommendations for future research.

\section{A framework examining the different viewpoints in the diffusion of broadband}

In order to understand and explain how a new technology is adopted many studies have used the theory of diffusion of innovations developed by Rogers (1995). According to his work an individual's decision to adopt a new technology is a process that occurs over a period of time and consisting of a series of actions and decisions rather than an instantaneous act. Roger's theory has been used for the study of several technologies (Rajagopal, 2002; Karahanna et al., 1999; Kumar and Swaminathan, 2003). It has subsequently been criticised for varying reasons (Elliot and Loebbecke, 2000; Kautz and Pries-Heje, 1996; Lyytinen and Damsgaard, 2001). For example, the theory is criticised for portraying diffusion as a simple linear process (Kautz and Pries-Heje 1996). It is argued that his view failed to sufficiently consider the relationship between suppliers and adopters and an active participation of potential adopters in the diffusion process. Beynon-Davies and Williams (2003) highlight the fact that technological diffusion is usually portrayed as a rational process but postulate that diffusion process is similar to broader social movements. Finally, Lyytinen and Damsgaard (2001) showed in their observations that complex technologies will not necessarily diffuse in a specific order.

The innovation diffusion theory as presented by Rogers implies that businesses would decide to adopt an innovation mainly because of its characteristics. This implies that other influences such as nature and size of business, background of business owners are disregarded. Additionally, different views, opinions and agendas of various groups involved in the process of the adoption of an innovation are not adequately represented in the theory of diffusion of innovation (Papazafeiropoulou et al., 2005). In this paper we agree that the other influences matter and we try to identify the different viewpoints that could be encountered in the diffusion process of broadband to SMEs. In doing this, we seek to identify possible gaps in perceptions that explains reasons for adoption or non-adoption and further explain perceived benefits if any. Thus we propose the use of a socio-technical approach such as social construction of technology (SCOT) (Pinch and Bijker, 1984) where the role of the various social groups in the production and use of an innovation is invaluable.

\subsection{Socio-technical approaches}

Socio-technical approaches criticize the assumption of linear models of innovation that the supply of a technology would create solutions that corresponded to user requirements, which could be diffused to potential adopters. Socio-technical approaches have in contrast shown that identifying current and future demand for technologies can be difficult (Klein and Kleinman, 2002; McLoughlin, 1999). 
Several research studies have used socio-technical approaches to unravel various phenomena in information systems. In Wilson and Howcroft (2002), Social shaping of technology (SST) has been used to study the failure of IS systems. They use SST concepts such as interpretative flexibility, translation, persuasion, stabilisation and irreversibility. The authors found SST to be particularly useful since it has as its core concern an understanding of the dynamics of the society-technology relationship. Their research suggested that it is not necessary for a technology to have changed in order for it to be perceived differently over time.

Social shaping of technology (SST) conceptualizes technology as socially shaped. According to Williams (1997), SST surfaced through the criticism of technological determinism. Technological determinism on the one hand presumes that particular paths of technological change are inevitable. SST studies on the other hand show that technology is a social product, which is patterned by its creation and use.

The origin of constructivist approach to technology can be found in the sociology of scientific knowledge (SSK) (McLoughlin, 1999). In social constructions, the nature of technology and what it can do are seen as a product of human interpretation.

SCOT provides a strong antidote to technological determinism. With SCOT, the development of a technological artefact is described as alternating between variation and selection, thereby resulting in a multidirectional model that contrasts linear models (Pinch and Bijker, 1984). The authors outline four components to the social construction of technology. They are: relevant social groups, interpretive flexibility, closure, and stabilization. In SCOT where the introduction of an innovation is faced with objections from different social groups, one seeks to find a solution that would make the innovation desirable for reasons that would supersede reasons for objections.

The point of SCOT is that the successful stages in the development of an artefact are not the only possible ones.

Steps involved in SCOT include:

- Identifying the relevant social groups

- Describing them in more detail

- Identifying the problems each of these groups has with respect to the artefact

- Identifying several variants of the solution around each of these problems.

This way of describing the developmental process brings out all the different kinds if conflicts. This model highlights the multidirectional character of a technological artefact. The interpretative flexibility of an artefact must be shown. In this case, the technological artefact is broadband and there are different social groups (SMEs inclusive) involved in its diffusion.

The interpretive flexibility principle of SST is useful where the need arises to understand how the problems and solutions associated with a technology present themselves differently to different social groups.

\subsection{Use of SCOT in information systems research}

SCOT has been utilized in several studies ranging from technological studies to information technology (IT) research. In a previous study of the relationship between IT and innovation in SMEs, Dierckx and Stroeken (1999), use SCOT in their study to determine the different views of various actors involved in car assembly firms. The authors created two scenarios to examine how car assembly firms decide on an order system for used parts. They then outlined the social spheres of influence affecting the scenarios and indicate the opinion of each 
relevant actor. Finally, analysis of the opinions of the various actors helped to determine the more advantageous scenario.

In this research, our model is based on the information systems variance model (Agarwal and Prasad, 1998) examining compatibility, complexity and relative advantage as the attributes that are of the most importance in Information Technology (IT) innovations. According to a study carried out by (Tornatzky and Klein (1982) only relative advantage, complexity and compatibility were found to be consistently related to the adoption of a technology. This model is nevertheless extended by identifying the various social groups involved in the production and use of the innovation.

In the model, an extension to the innovation diffusion and information systems variance model is proposed by identifying the different social groups involved in the diffusion of broadband as described in SCOT. We could then obtain the views of the different social groups in relation to the relative advantage, compatibility and complexity part of the innovation diffusion model. However, SCOT provides no defined way of identifying the social groups (Pinch and Bijker, 1984). The research would therefore use guidelines prescribed in stakeholder research. This is because stakeholders are similar to social groups. Pouloudi and Whitley (1997) highlighted the fact that in previous stakeholder research, there have not been any specifications as to how stakeholders can be identified. They highlight the inherent problems that arise out of the lack of a systematic stakeholder identification approach such as the fact that generic stakeholder lists are not applicable in every context. They would therefore fail to identify all relevant information systems stakeholders because stakeholder groups such as the systems' developers and users are not usually included in the generic stakeholder lists. Finally, the authors prescribe a method of stakeholder identification which will be used to identify the social groups which are involved in the diffusion of broadband to SMEs in this study.

\subsection{Identifying the social groups using stakeholder identification}

Pouloudi and Whitley (1997) base the identification of stakeholders on four underlying principles. They are;

- Stakeholders depend on the specific context and time frame

- Stakeholders cannot be viewed in isolation

- The position of each stakeholder may change over time

- Feasible options may differ from the stakeholders' wishes

The authors suggest all these principles be put in use when attempting to identify stakeholders in order to understand organizational and inter-organizational reality, to explain past circumstances and to use the conclusions to plan for future activity in a realistic manner.

In our model, we propose to extend the innovation diffusion model by identifying the different social groups involved in the diffusion of broadband as described in SCOT. We also use interviews to obtain the views of the different social groups in relation to the technological characteristics of the innovation. The social groups involved in the diffusion of broadband to SMEs, as illustrated in figure 1, include: the SMEs, the government (Choudrie et al., 2003, Office of the e-Envoy, 2001), professional associations (Intellect, 2003), Independent bodies striving for broadband adoption (BSG, 2004), private consultants (Philpott, 2004) and vendors (Cisilion, 2002); (BTOpenworld, 2002). 


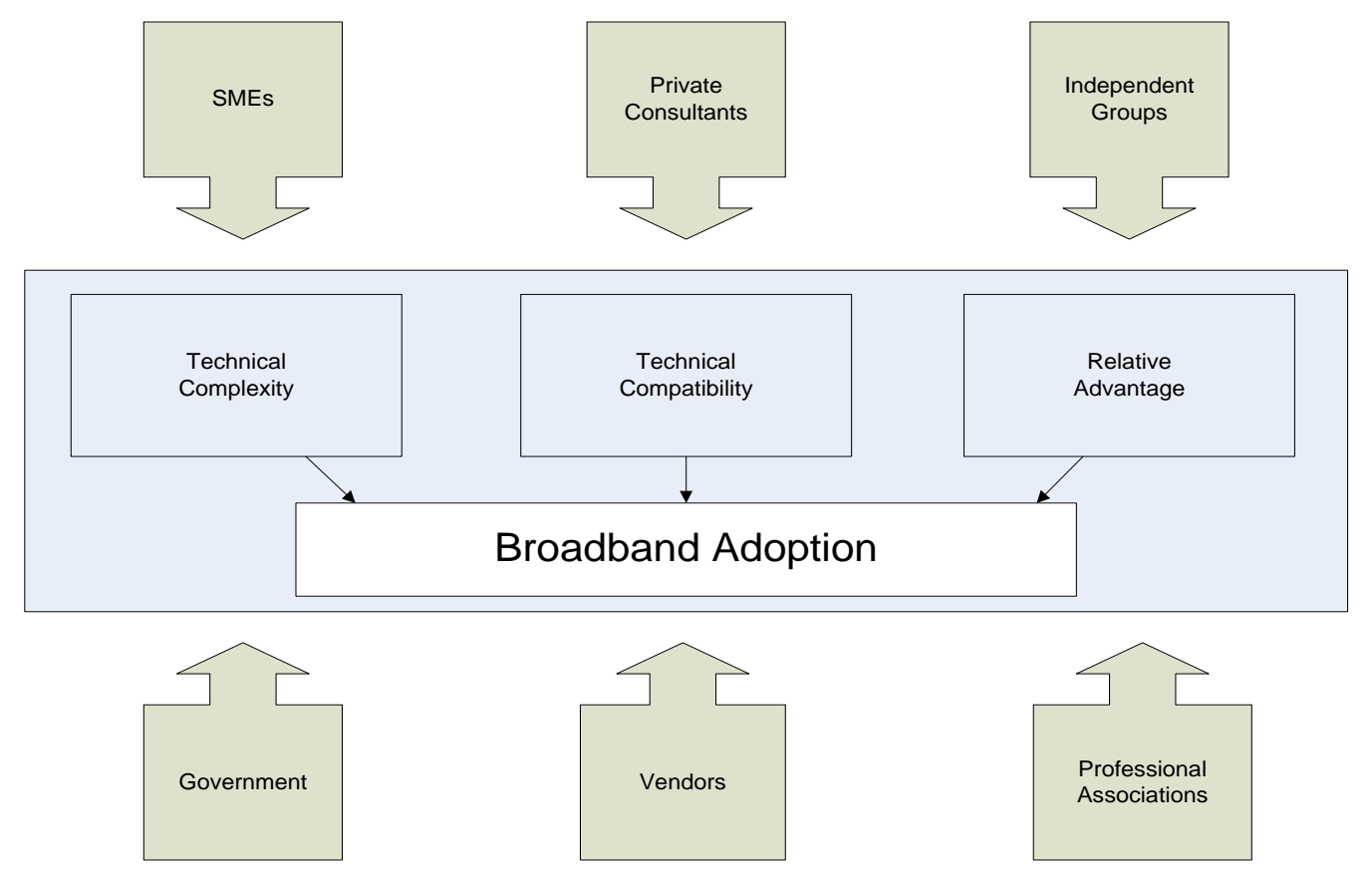

Figure 1A framework examining the different viewpoints in broadband adoption

In the next section we discuss the methodology employed in this research. Then we examine and analyse the different viewpoints that shape the diffusion of broadband using the UK as an example of a dynamic broadband market.

\section{Methodology}

This research adopts an interpretive stance. We do this in order to reflect the researchers' efforts to identify multiple social groups and their interpretations.

Information systems research can be classified as interpretive if it is assumed that our knowledge of reality is gained only through social constructions such as language, consciousness, shared meanings, documents, tools and other artefacts. Interpretive research does not predefine dependent and independent variables, but focuses on the complexity of human sense making as the situation emerges (Kaplan and Maxwell, 1994). Interpretive research methods aim at the "understanding of the context of the information system and the process whereby the information system influences and is influenced by the context" (Walsham, 1993) (p. 4-5).

This paper explores broadband diffusion to SMEs using data obtained from interviews conducted in England in 2006. Using available literature, we identified the relevant social groups involved in broadband diffusion. Of these groups, we then selected representatives to collect empirical data. We interviewed fifteen SMEs, one vendor, one private consultant and one employee of a government agency. Twelve of the SMEs that were selected are currently using broadband. Some of these used dial-up previously while others have only used broadband for Internet access. The SMEs businesses range from furniture sales, to estate agents, Internet cafes, uniform wholesalers, training institutions and travel agents. The reason for selecting such SMEs was to represent the various categories of industries that SMEs can be involved in.

The interviews were conducted between April and May 2006 and lasted between 15-40 minutes. In each meeting we used an interview guide which contained 9 questions for each of the social groups. These questions covered themes relating to the diffusion attributes previously mentioned and were open ended to glean more information than was anticipated.

This is a pilot study that will be further examined in future research. In the next section we present an analysis of the different views obtained from the field work. 


\section{Analysis of broadband diffusion}

As we mentioned previously, the various groups associated with the diffusion of broadband, as illustrated in figure 1, have differing views about its attributes. These views are presented below.

\subsection{Compatibility}

This attribute is the degree to which broadband, as an innovation, is seen to be consistent with existing values, needs and past experiences of SMEs as the potential adopters. The vendor like the interviewee from the government agency felt that all SMEs would find broadband integral to running their businesses. In contrast however, in the private consultant's opinion, broadband would not suit all SMEs and "would only be of benefit with suitable training in the advantages the Internet can bring to a business." The vendor said that broadband is easy to install and integrate into SMEs existing systems "Installation of our services is very simple. With plug and play devices the setup process would be completed within about 2 minutes. There are very little details that are customer specific so the human intervention is limited". Similarly the interviewee from the government agency said that: "Hardware is cheap and provided you get the right support you really shouldn't have any problems using broadband". For some of the SMEs however, they thought having broadband installed would be too costly for them. Some SMEs felt broadband was very useful as it helped to save money on the cost of communication. For example, a furniture supplier used broadband connection to send and receive invoices and make online orders for stock while another furniture supplier said he had no use for broadband. This shows some disparity in the views of the social groups regarding the compatibility attribute of broadband.

\subsection{Complexity}

This is described as the degree to which broadband as an innovation is perceived as relatively difficult to understand and use. The interviewee from the government agency opined that all SMEs would benefit from using broadband. In his words "Competition is using broadband and so should you. You should do it to keep up with them or they'll take your business away." However, he felt that using broadband would create some adverse issues for the SMEs: "they have to develop new skill set such as using the internet to transfer files and things like voice over IP as well". The vendor in contrast thought that using broadband was quite easy and that "SMEs would wonder why they waited so long to use it". Some of the SMEs were quite happy using broadband while one of the SMEs was experiencing a problem with his broadband connection while the interview was going on and did not know what to do about it. Another SME, which had previously used a different vendor, had a problematic service for 3 months and did not know what to do at such times. The interviewee said: "Unfortunately, the connection wasn't working properly and despite several attempts to contact the company we did not get them to fix it and that messed up business for 3 months". Again, the social groups do not view complexity of broadband in the same light.

\subsection{Relative advantage}

This is described as the degree to which an idea which an innovation is perceived as being better than the idea it supersedes and is usually expressed in terms of economic profitability, social prestige or other benefits. The vendor says that broadband in better than dial up. Similarly, the interviewee from the government agency agreed that broadband is definitely better than dial-up because "it is flat rate and a lot quicker". He however raised the issue of susceptibility to viruses: "They will have to deal with the fact that using broadband will make them more vulnerable to viruses and privacy issues as well". The private consultant also thought that broadband was better but that economic benefits would not emerge immediately. "If they have been using dial-up, very easy if it's a new user to the internet there is probably a 12 month period until usage is optimised". The SMEs that used dial-up previously also agree that broadband is better than dial-up. Unlike the other attributes, there is a general consensus on the relative advantage attribute of broadband. Apart from the security issues raised by the 
interviewee from the government agency, the social groups agree that broadband is better than dial-up.

In the next section, we discuss the results from this analysis.

\section{Discussion}

In this research, the views that have been sourced from the social groups show the differences in the way broadband is perceived. The innovation is seen as the next big thing by both the vendors and the government. As a result several government policies and initiatives have been aimed at broadband diffusion (BSG, 2004). From the data collected, the SMEs do not share these views and it does not seem that they have been impacted by these initiatives. Training was a point that was raised by the private consultant. He said the SMEs would not realise economic gain from using broadband if they were not trained on the advantages it could give. The SMEs that were interviewed had not received any funding nor had they received any training on the advantages using broadband could give. This was evident among the SMEs even though they were using broadband, they only used it to send and receive mails and a few used it to upload and send pictures but only one of them had considered trading online and only two were using VoIP. This leads to the conclusion that broadband has not necessarily changed the way the Internet is used. Further investigation will be necessary to determine whether more SMEs would adopt broadband if they knew all they could use it for and how beneficial, in economic terms, it could be.

Broadband providers, the UK government and other interest groups have continually stressed the importance of broadband but with little results where SMEs are concerned. There are many factors that could influence SMEs in deciding whether or not they would adopt a new technology such as broadband. In this research, we have considered three main innovation attributes of broadband and have looked at the different social groups and their views about these attributes.

Relative advantage seems to be the only attribute where there is a general consensus among the social groups. Nevertheless the government agency employee raised the issue of security because SMEs susceptibility would be heightened with the use of broadband. On the issue of compatibility and complexity the SMEs that have adopted broadband use it to send and receive mails. A few others use it to make purchases. However, the vendor and the government agency interviewee say broadband provides several other advantages such as flexibility to change existing practices, target new customers, greater productivity, improvement in business processes, and greater independence while allowing them to take advantage of associated technologies. The SMEs seem to be unaware of these advantageous uses.

We analysed the perceived attributes of broadband and the different views of the social groups involved. We argued that the perception gaps between different social groups could explain reasons for adoption or non-adoption. Our initial findings show that in the case of broadband adoption and diffusion it seems that there is no consensus as to how the technology is useful, cheap or convenient for SMEs to use. There are a number of assumptions taken by the vendors and the government about broadband adoption that seem to not be shared by SMEs. This seems to be mainly due to a lack of awareness from the SMEs about the benefits of broadband and its opportunities for e-business. Our research showed that was one issue that needs to be further analysed.

The vendor raised the issue of awareness and this has been mentioned by the interviewee from the government agency but the SMEs that have adopted broadband did not do so as a result of awareness programmes aimed at them. Some other issues came to light which were not included in the framework guiding this research and they are equally related to lack of awareness. Such issues include cost and funding, full use, quality of service and access.

The cost of obtaining broadband was a recurring issue in the interviews conducted. Many of the SMEs that had not adopted broadband said it was an unnecessary expense. The 
government agency interviewee said that cost might have been a deterrent originally but that the cost of obtaining broadband has reduced significantly in recent times. While the vendor was of the opinion that as a result of costs, SMEs might want to cut corners: "Some users are initially looking for the cheapest package, but don't realise that they are losing quality". The cost of obtaining broadband would also require further investigation because in the literature review the government and vendors all say it would save money on the long run. If SMEs were shown how broadband could save them money then their response could possibly be different

The government agency interviewee said that funding was available for SMEs investing in broadband but was unavailable in some urban areas. However, the SMEs that were interviewed said they had received no funding and were unaware of any such initiatives.

Previously, in section five, we mentioned that there are several advantageous uses for broadband. These include opportunities for e-business, efficient data back-up, video conferencing and Voice over Internet Protocol (VoIP). The SMEs that were interviewed have been using broadband to send and receive mails while a few others use it to make purchases. This shows that SMEs are not making full use of broadband.

Another issue that came up is quality of service. The vendor mentioned quality as a reason to select a broadband provider carefully. Some of the SMEs that were interviewed have experienced poor quality of service from their broadband providers. One of the SMEs was experiencing a problem with his broadband connection while the interview was going on and did not know what to do about it. Another SME, which had previously used a different vendor, had an epileptic service for 3 months and did not know what to do about it either. Such experiences would possibly deter other SMEs who would have otherwise decided to adopt broadband.

\section{Conclusions/Further research directions}

We have looked at broadband and its adoption by SMEs in the UK. We presented our research approach and discussed the framework using fieldwork where representatives of the identified social groups were asked to offer their opinions, which we analysed to get a better understanding of broadband diffusion to SMEs. This led us to the conclusion that broadband has not necessarily changed the way the Internet is used.

Considering the usefulness and inherent limitations of the diffusion of innovations theory, we developed a model based on the first three innovation attributes (Agarwal and Prasad, 1998); (Cooper and Zmud, 1990); (Crum et al., 1996). This model uses innovation attributes and takes into consideration the various social groups and views that are related to broadband uptake by SMEs using SCOT.

We started by identifying the relevant social groups and have made an initial attempt to see their viewpoints. The UK government set out to have the most extensive and competitive broadband adoption in the G7 by 2005 and sees broadband as the next leap forward for the internet (BSG, 2004). They expect SMEs in particular to benefit from its adoption. From the data we have collected the social groups have differing view points on the issue of broadband diffusion. The SMEs that have adopted broadband have done so for communication purposes and the SMEs that have not adopted feel they have no use for it but the government and vendors think there are numerous beneficial uses. This leads to the conclusion that broadband has not necessarily changed the way the internet is used. On the issue of costs as well, funding is available but none of the SMEs interviewed have benefited from it. Drawing from one of the elements of SCOT (Pinch and Bijker, 1984), closure can only be achieved if the government and vendors educate the SMEs about the benefits of broadband. The SMEs also have to be informed about the types of services provided by vendors and the initiatives available to them should they decide to adopt broadband. Then the SMEs can make an informed choice to adopt or reject. 
Future research will include consideration of awareness under which we will look at cost, full use and quality of service and the perceptions of the social groups on these issues. Further research would also require investigation into the kind of funds available to SMEs and how the government agencies decide which SMEs get funded. We will be investigating whether the lack of full use is due to a lack of awareness and if training will improve the use possibly resulting in a better uptake of the technology thereby providing opportunities for expansion and growth through e-business.

We believe that our research could be useful for SMEs considering adoption of new technologies such as broadband as well as policy makers that seek to apply effective technological adoption policies as well as researchers in the field of innovation diffusion.

\section{References}

Agarwal, R. and Prasad, J. (1998) A conceptual and operational definition of personal innovativeness in the domain of information technology., Information Systems Research, 9,2 204-215.

Beynon-Davies, P. and Williams, M. D. (2003) The Diffusion of Information systems Development Methods, Journal of Strategic Information Systems, 1229-46.

BSG (2004) Third Annual Report and Strategic Recommendations. accessed 25/10/ 2004 online at http://www.intellectuk.org/publications/reports/BSG_3rd_annual_report.pdf

BTOpenworld (2002) BT Openworld shows businesses the route to high-speed cost savings accessed 17/10/2005 online at http://www.cisilion.com/bnews_benefits.htm

Chappell, C.,Feindt, S. and Jeffcoate, J. (2002) Best practice in SME adoption of ecommerce, Benchmarking: An International Journal, 9,2 122-132.

Choudrie, J.,Papazafeiropoulou, A. and Lee, H. (2003) A web of stakeholders and strategies: a case of broadband diffusion in South Korea, Journal of Information Technology, 18,4 281-291.

Cisilion (2002) Small to medium businesses can't see broadband benefits accessed 18/11/ 2004 online at http://www.cisilion.com/bnews_intellect.htm

Cooper, R. B. and Zmud, R. W. (1990) Information Technology Implementation Research: A Technological Diffusion Approach, Management Science, 36,2 123.

Crum, M. R.,Premkumar, G. and Ramamurthy, K. (1996) An assessment of motor carrier adoption, use, and satisfaction with EDI, Transportation Journal, 35,4 44.

Dierckx, M. A. F. and Stroeken, J. H. M. (1999) Information technology and innovation in small and medium-sized enterprises, Technological Forecasting and Social Change, 60,2 149-166.

Office of the e-Envoy, (2001) UK Online: The Broadband Future

Elliot, S. and Loebbecke, C. (2000) "Interactive, inter-organisational innovations in electronic commerce." Information Technology and People, 13,1 46-66.

Firth, L. and Kelly, T. (2001) Broadband briefing paper, ITU, Geneva accessed 23/5/ 2005 online at http://www.itu.int/osg/spu/ni/broadband/workshop/briefingpaperfinal.doc

Intellect (2003) The Benefits of Broadband to SMEs in the ICT Sector Interim Conclusions from Intellect Member Research accessed 6/4/ 2005 online at http://www.intellectuk.org/campaigns/broadband/benefits/Exec_summary_Int ellect.pdf 
Jeffcoate, J.,Chappell, C. and Feindt, S. (2002) Best Practice in SME Adoption of Ecommerce, Benchmarking: An International Journal, 9,2 122-132.

Kalakota, R. and Robinson, M. (2001) E-Business 2.0: Road-map for Success,, Addison-Wesley, Harlow.

Kaplan, B. and Maxwell, J. A. (1994) Qualitative Research Methods for Evaluating Computer Information Systems. In Evaluating Health Care Information Systems: Methods and Applications(Eds, Anderson, J. G., Aydin, C. E. and Jay, S. J.) Sage publications, Thousand Oaks CA.

Karahanna, E.,Straub, D. W. and Chervany, N. L. (1999) Information Technology Adoption Across Time: A Cross-sectional Comparison of pre-adoption and post-adoption Beliefs, MIS Quarterly, 23,2 183-214.

Kautz, K. and Pries-Heje, J. (1996) Diffusion and adoption of information technology, Chapman \& Hall, London.

Klein, H. K. and Kleinman, D. L. (2002) The Social Construction of Technology: Structural Considerations, Science, Technology and Human Values, 27,1 2852.

Kumar, S. and Swaminathan, J. M. (2003) Diffusion of Innovations Under Supply Constraints, Operations Research, 51,6 866.

Levy, M.,Powell, P. and Yetton, P. (2002) The dynamics of SME information systems, Small Business Economics, 19,4 341.

Lyytinen, K. and Damsgaard, J. (2001) The role of intermediating institutions in the diffusion of electronic data interchange (EDI): How industry associations intervened in Denmark, Finland, and Hong Kong, Information Society, 17,3 195.

McLoughlin, I. (1999) Creative technological change: The shaping of technology and organisations In The management of technology and innovation(Ed, Preece, D.) Routledge, London and New York.

Merlin, B. (2004) Internet Marketing in Exports- A Useful Tool In Small Businesses, Small Enterprise Development, 15,4 38-44.

Papazafeiropoulou, A.,Gandecha, R. and Stergioulas, L. 2005 Interpretive flexibility along the innovation decision process of the UK NHS care records service (NCRS). Insights from a local implementation case study In Proceedings of the Thirteenth European Conference on Information Systems Regensburg, Germany

Philpott, M. (2004) Ovum white paper: DSL Services for Small Enterprises

Pinch, T. J. and Bijker, W. E. (1984) The Social Construction of Facts and Artefacts: Or How the Sociology of Science and the Sociology of Technology Might Benefit Each Other In The Social Construction of Technological Systems(Eds, Bijker, W. E., Hughes, T. P. and Pinch, T. J.) MIT Press, Massachusetts Institute of Technology, pp. 18-50.

Pouloudi, A. and Whitley, E. A. (1997) Stakeholder identification in interorganizational systems: gaining insights for drug use management systems, European Journal of Information Systems, 6,1 1-14.

Rajagopal, P. (2002) An innovation-diffusion view of implementation of enterprise resource planning (ERP) systems and development of a research model, Information \& Management, 40,5 87.

Rogers, E. M. (1995) Diffusion of innovations, Free Press, New York.

Saban, K. A. and Rau, S. E. (2005) The Functionality of Websites as Export Marketing Channels for Small and Medium Enterprises, Electronic Markets, 15,2 128-135. 
Sandler, M. and Boggs, R. (2001) Small business eCommerce- Paving the Way for the Next Generation of Opportunity accessed 15/10/ 2004 online at http://netobjects.com

Tornatzky, L. G. and Klein, K. J. (1982) Innovation Characteristics and Innovation Adoption-Implementation: A Meta Analysis of Findings, IEEE Transactions on Engineering Management, 29,11 28-45.

Walsham, G. (1993) Interpreting Information Systems in organisations, Chichester.

Williams, R. (1997) The social shaping of information and communication technologies In The social shaping of information superhighways(Ed, Kubiceck, H., Dutton, W.H., Williams, R.) Campus Verlag/St. Martin's Press, New York.

Wilson, M. and Howcroft, D. (2002) Re-conceptualising failure: Social shaping meets IS research, European Journal of Information Systems, 11,4 236. 